\title{
Atypical metastasis of renal cell carcinoma to the uvula: case report and review of literature
}

This article was published in the following Dove Press journal: International Medical Case Reports Journal

\section{Parth Khade \\ Srinivas Devarakonda}

Louisiana State University Health, Shreveport, LA, USA
Correspondence: Srinivas Devarakonda Louisiana State University Health, New I50I Kings highway, Shreveport, LA, USA 71105

Tel +| 3188131496

Email sdeval@|suhsc.edu

\begin{abstract}
Renal cell carcinoma (RCC) is a common malignancy with high metastatic potential, primarily due to its extensive vascularity. Common sites of metastasis include lungs, bone, lymph nodes, liver, and brain. However, rare cases of metastasis to other sites including inguinal lymph nodes, peritoneum/mesentery, and orbit have been published in the literature. Herein, a unique case involving metastasis of RCC to the uvula is presented. The patient is a 55-year-old White female with a past medical history of stage 3 (T3aN0M0) RCC s/p nephrectomy 3 years prior to presentation. She had symptoms of a foreign body sensation at the back of her throat, and oropharyngeal examination revealed uvular erythematous mass with vascularity. Uvular biopsy and complete excision were performed, which revealed metastatic RCC. Palate biopsy was negative and revealed only squamous mucosa with mild chronic inflammation. To our knowledge, there is only one other documented case of RCC metastasis to the uvula in the literature. Keywords: kidney cancer, soft tissue, cancer, metastasis, vascular, palate
\end{abstract}

\section{Introduction}

Renal cell carcinoma (RCC) is a common malignancy with high metastatic potential, primarily due to its extensive vascularity. In 2017 , there were $\sim 64,000$ estimated new cases of RCC in the US (representing 3.8\% of all new cancer cases), resulting in 14,400 estimated deaths. Approximately 9,500 of these estimated deaths were noted to be in males, while $\sim 5,000$ were in females. ${ }^{1}$ Globally, RCC accounts for $\sim 2 \%$ of all malignancies, with $\sim 189,000$ new cases annually. In the US, epidemiological data have shown that the incidence rates are higher among the African Americans compared to Caucasians. Established risk factors for RCC include tobacco, obesity (as measured by BMI), chronic hepatitis $\mathrm{C}$, ionizing radiation, and end stage renal disease (ESRD). ${ }^{2}$ Histopathological subtypes of RCC include clear cell $(60 \%-70 \%)$, papillary $(5 \%-15 \%)$, chromophobic $(5 \%-10 \%)$, oncocytic $(5 \%-10 \%)$, and collecting duct $(<1 \%){ }^{3}$ Common sites of metastasis include lungs, bone, lymph nodes, liver, and brain. However, there have been rare cases of metastases to other sites including inguinal lymph nodes, peritoneum/mesentery, and orbit published in the literature. ${ }^{4-6}$ Herein, a rare case involving metastasis of RCC to the uvula is presented.

\section{Case presentation}

This case report is about a 55-year-old White female with a PMH of RCC diagnosed 3 years prior to presentation with uvular metastasis. She was noted to have stage 3 RCC at the time of presentation (T3aN0M0). She had renal ultrasound that showed large $9.2 \mathrm{~cm}$ 
solid right renal mass without hydronephrosis and underwent right nephrectomy that revealed grade 3 clear cell carcinoma with extension of the mass into the renal sinus fat and some vascular invasion into the renal sinus, without renal vein invasion. Approximately 1 year after nephrectomy, computed tomography (CT) showed small nodule along the anterior aspect of the uncinate process of the pancreas, and biopsy revealed grade 2 metastatic RCC. Celiac axis lymph nodes were negative, and the patient underwent the Whipple procedure.

A year later, the patient reported having persistent headaches, which started $\sim 3$ months prior to visit. She had also reported having vertical diplopia on upward gaze. CT of the head and sinuses was taken, which revealed a $2.2 \mathrm{~cm}$ circumscribed mass of the left ethmoid sinus. Magnetic resonance imaging (MRI) was taken for further evaluation, which showed a well circumscribed, intensely enhancing mass $2.3 \mathrm{~cm}$ in diameter in the left nasal cavity, bulging into the left maxillary sinus. Stereotactic CT-guided endoscopic sinus surgery was performed, which showed that the mass extended into the maxillary sinus and was causing a dehiscent lamina papyracea. A large maxillary antrostomy and sphenoidectomy was required to resect the tumor completely. A septal perforation was visualized which was thought to be from extension of the tumor in the nasal cavity onto the septum. Embolization was performed by interventional radiology prior to endoscopic sinus surgery and resulted in successful preoperative embolization of bilateral internal maxillary arteries. Frozen sections were sent to pathology and confirmed RCC of the left ethmoid and nasal cavity. Following surgical resection of ethmoid mass, the patient underwent intensity-modulated radiation therapy to the ethmoid sinus area via tomotherapy for a total dose of 3,900 cGy. Chemotherapy treatment included sunitinib, which was stopped based on the predicted lack of response on molecular profiling test results, and later everolimus, which was stopped due to grade 3 fatigue and severe mucositis.

Approximately a year after left ethmoid sinus metastasectomy, metastasis to the uvula was detected. The patient presented with vague nasal symptoms such as congestion, and MRI of the sinuses was negative for any tumor. Examination of the oropharynx by otorhinolaryngology revealed a uvular erythematous mass with vascularity, $\sim 2 \mathrm{~mm}$ in size, and a $2 \mathrm{~mm}$ papillomatous lesion in the left hard palate. Positron emission tomography/CT looking for the extent of disease at this time showed no focal fluorodeoxyglucose (FDG) avid abnormalities in the region of the uvula, but did show moderately FDG avid $1.2 \mathrm{~cm}$ noncalcified nodule medially in the left suprahilar region, later found on biopsy to be bronchial carcinoid. Uvular biopsy and complete excision were performed by otorhinolaryngology surgery, which revealed metastatic RCC (Figure 1). Palate biopsy was negative and revealed

\section{A}

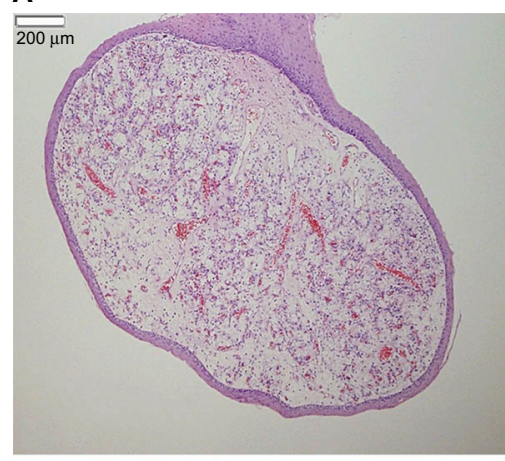

C

\section{B}

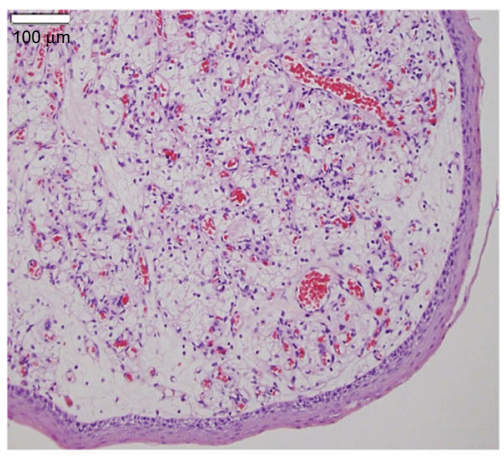

D

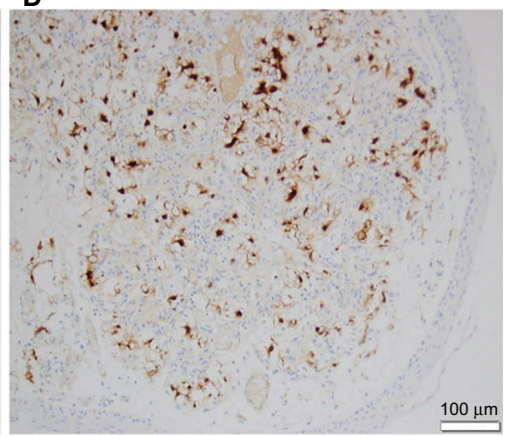

Figure I Slides of metastatic renal cell carcinoma (RCC) to the uvula.

Notes: Submucosal clear cell RCC with overlying normal squamous epithelium, (A) H\&E 4X and (B) H\&E I0X. Cytoplasmic and membranous staining of tumor cells with RCC antibody by immunohistochemistry, (C $4 \times$ and (D) $10 \times$. 
only squamous mucosa with mild chronic inflammation. The patient was restarted on a lower dose of everolimus, which she tolerated well without experiencing any of the previously mentioned side effects. Everolimus was continued until recent disease progression with new lung metastases visualized on $\mathrm{CT}$, at which time the patient was switched to pazopanib, an oral vascular endothelial tyrosine kinase inhibitor.

\section{Copyright/ethics}

Written informed consent has been provided by the patient to have the details of her case and accompanying image published.

\section{Discussion}

$\mathrm{RCC}$ originates from the renal cortex and comprises the majority of primary renal malignancies. Classically, RCC presents with flank pain, hematuria, and a palpable renal mass; however, this only occurs in a minority of patients with RCC and typically indicates the presence of an advanced disease state. Our index case presented with complaints of flank pain, which prompted renal ultrasound that showed a solid renal mass, with nephrectomy revealing a stage 3 (T3aN0M0) carcinoma at presentation. Paraneoplastic symptoms involving RCC can include anemia, hypercalcemia, fever, hepatic dysfunction, and erythrocytosis. Initial radiographic testing includes either CT or ultrasound, and MRI can also be utilized to determine the involvement of inferior vena cava.

This case gives a unique presentation of RCC metastasizing to the uvula, which differs from more common areas of metastasis including lungs, brain, bone, lymph nodes, and liver. Metastasis of RCC to the paranasal sinuses is rare; however, there have been multiple documented case reports describing RCC spread to the nasal septum as well as to the maxillary and ethmoid sinuses. ${ }^{7}$ RCC is the most common malignancy that metastasizes to this region. ${ }^{8,9}$ The most common path via which RCC cells spread to the sinonasal region includes that via the inferior vena cava-heart-maxillary artery as well as via the avalvular vertebral venous plexus and the intracranial venous plexus. ${ }^{9}$ The most common sinonasal sites of metastasis include the maxillary sinuses (36\%), ethmoid sinuses $(25 \%)$, frontal and sphenoid sinuses $(17 \%)$, and the nasal cavity $(11 \%) .{ }^{9}$ Of note, in our index case, metastasis of RCC to the ethmoid sinus was seen (and surgically resected) prior to the detection of RCC metastasis to the uvula. There have also been multiple documented cases and literature reviews of RCC metastasis to the tongue. ${ }^{10}$ To our knowledge, there is only one other documented case of RCC metastasis to the uvula in the literature. ${ }^{11}$
Metastasis of RCC to the head and neck region is rare. ${ }^{12,13}$ Sinonasal malignancies are typically primary neoplasms, with metastatic tumors being rare; however, RCC is the most common malignancy to metastasize to this region. An analysis of 412 metastatic lesions to the oral soft tissues showed that RCC mainly metastasized to the tongue compared to the other oral soft tissues, with a total of 19 cases. ${ }^{14}$ However, the oral cavity in general is an uncommon site for metastasis, as only $1 \%$ of all malignant oral tumors are composed of metastatic lesions. A literature review performed by Azam et $\mathrm{l}^{12}$ revealed a total of 28 cases that presented with tongue metastases prior to the diagnosis of primary RCC. This is in part due to the fact that metastatic lingual lesions present with ulcerating, polypoid, or vegetating lesions similar to primary malignancies of the tongue. ${ }^{12}$ Thus, in cases in which an oral ulcerating lesion is seen, it is always important to make histopathological diagnosis instead of clinical diagnosis based on appearance, as the prognosis and treatment will vary significantly based on the type of malignancy. This is also particularly true for masses presenting with a hypervascular structure, and preoperative diagnosis can allow for the preparation for increased bleeding during surgical excision. In our index case presentation, the diagnosis of RCC was made $\sim 2$ years prior to the detection of metastatic ethmoid mass and $\sim 3$ years prior to the detection of the metastatic uvular mass. Possible routes of spread include arterial, venous, and lymphatic circulation.

In a review performed by Hirshberg et $\mathrm{al}^{15}$ analyzing 157 cases of metastases to the oral mucosa, there were a total of 21 cases of metastatic RCC. Out of these 21 cases, only two had metastasized to the palate as compared to other oral sites, and there were no documented cases of metastases to the uvula, as was seen in our index case.

RCC metastasis to the head and neck region including the paranasal sinuses and the lingual area also typically predicts poor survival, and the treatment can include surgical excision of metastatic lesions and radiotherapy for palliation of pain, control of bleeding, and management of dysphagia. This is particularly important for larger metastatic lesions, as continued metastatic tumor enlargement can potentially lead to airway obstruction. In our index case, surgical excision was used to remove the uvular mass after being detected on MRI. Adjuvant radiation therapy can also be used with palliative intent. In our index case, surgical resection was followed by intensity-modulated radiation therapy to the ethmoid sinus area via tomotherapy, with a total dose of 3,900 cGy. Embolization of vascular lesions seen in certain head and neck tissues (including the tongue) has been discussed and 
was utilized in our index case for embolization of internal maxillary arteries prior to endoscopic sinus surgical excision of ethmoid mass, but was not required prior to surgical excision of the uvular metastatic lesion.

\section{Acknowledgment}

The authors like to thank Dr Ashley Flowers, Department of Pathology, Louisiana State University Health, for providing the histopathology figures for the manuscript.

\section{Disclosure}

The authors report no conflicts of interest in this work.

\section{References}

1. Siegel RL, Miller KD, Jemal A. Cancer statistics, 2017. CA Cancer J Clin. 2017;67(1):7-30.

2. Lindblad P. Epidemiology of renal cell carcinoma. Scand J Surg. 2004;93(2):88-96.

3. Rini BI, Campbell SC, Escudier B. Renal cell carcinoma. Lancet. 2009;373(9669):1119-1132.

4. Chaudhry QS, Bhatty TA, Khan Z, Osman EM. Renal cell carcinoma: atypical metastasis to inguinal lymph nodes. Urol Ann. 2017;9(1):80-82.
5. Staderini F, Cianchi F, Badil B, et al. A unique presentation of renal clear cell carcinoma with atypical metastases. Int J Surg Case Rep. 2015;11: 29-32.

6. Sountoulides P, Metaxa L, Cindolo L. Atypical presentations and rare metastatic sites of renal cell carcinoma: a review of case reports. $J$ Med Case Rep. 2011;5:429.

7. Singh J, Baheti V, Yadav SS, Mathur R. Occult renal cell carcinoma manifesting as nasal mass and epistaxis. Rev Urol. 2014;16(3):145-148.

8. Ziari M, Shen S, Amato RJ, The BS. Metastatic renal cell carcinoma to the nose and ethmoid sinus. Urology. 2006;67(1):199.

9. Ralli M, Altissimi G, Turchetta R, Rigante M. Metastatic renal cell carcinoma presenting as a paranasal sinus mass: the importance of differential diagnosis. Case Rep Otolaryngol. 2006;2017:9242374.

10. Lang EE, Patil N, Walsh RM, Leader M, Walsh MA. A case of renal cell carcinoma metastatic to the nose and tongue. Ear Nose Throat J. 2003;82(5): 382-383.

11. Lansigan NC Jr, Benisch BM, Sidoti JS. Renal cell carcinoma presenting as metastasis to the uvula. Urology. 1973;2(4):449-451.

12. Azam F, Abubakerr M, Gollins S. Tongue metastasis as an initial presentation of renal cell carcinoma: a case report and literature review. J Med Case Rep. 2008;2:249.

13. Altuntas O, Petekkaya I, Suslu N, Gullu I. Renal cell carcinoma metastatic to the tongue: a case report and review of the literature. J Oral Maxillofac Surg. 2015;73(6):1227-1230.

14. Irani S. Metastasis to the oral soft tissues: a review of 412 cases. $J$ Int Soc Prev Community Dent. 2016;6(5):393-401.

15. Hirshberg A, Leibovich P, Buchner A. Metastases to the oral mucosa: analysis of 157 cases. J Oral Pathol Med. 1993;22(9):385-390.
International Medical Case Reports Journal

\section{Publish your work in this journal}

The International Medical Case Reports Journal is an international, peer-reviewed open-access journal publishing original case reports from all medical specialties. Previously unpublished medical posters are also accepted relating to any area of clinical or preclinical science. Submissions should not normally exceed 2,000 words or

\section{Dovepress}

4 published pages including figures, diagrams and references. The manuscript management system is completely online and includes a very quick and fair peer-review system, which is all easy to use. Visit $\mathrm{http}: / /$ www.dovepress.com/testimonials.php to read real quotes from published authors.

Submit your manuscript here: https://www.dovepress.com/international-medical-case-reports-journal-journal 